\title{
High-NA-based virtual point detectors for photoacoustic imaging
}

Changhui Li, Lihong V. Wang

Changhui Li, Lihong V. Wang, "High-NA-based virtual point detectors for photoacoustic imaging," Proc. SPIE 7177, Photons Plus Ultrasound: Imaging and Sensing 2009, 71770R (24 February 2009); doi: 10.1117/12.808514

SPIE. Event: SPIE BiOS, 2009, San Jose, California, United States 


\title{
High-NA-based virtual point detectors for photoacoustic imaging
}

\author{
Changhui Li and Lihong V. Wang \\ Optical Imaging Lab, Department of Biomedical Engineering \\ Washington University in St. Louis, St. Louis, MO, 63130
}

\begin{abstract}
We demonstrated the focal point of a high-numerical-aperture (NA) ultrasonic transducer can be used as a virtual point detector. This virtual point detector detects omnidirectionally over a wide acceptance angle. It also combines a large active transducer surface and a small effective virtual detector size, thus the sensitivity is high compared with that of a real point detector, and the aperture effect is small compared with that of a finite size transducer. Phantom experiments are provided to demonstrate the applications of high-NA-based virtual point detectors in photoacoustic tomography and thermoacoustic tomography. The virtual point detector is also used to image the cerebral cortex of mice.
\end{abstract}

Keywords: photoacoustic tomography,thermoacoustic tomography, virtual point detector

\section{INTRODUCTION}

Photoacoustic (PA) tomography (PAT) is a promising non-ionizing, non-invasive biomedical imaging modality. ${ }^{1,2}$ It detects ultrasound signals generated by the thermal expansion processes after the radiation energy of electromagnetic (EM) wave is deposited as heat in the light-absorbing tissue. The radiation sources used in PAT generally includes laser or radio-frequency (RF) wave pulses, as well as the intensity modulated continuous waves $(\mathrm{CW})$. When using RF waves, PAT is also refers to the thermoacoustic tomography (TAT). PAT combines the sensitive optical contrast and high ultrasonic resolution, and has been successfully applied in imaging both small animal and human tissues. ${ }^{3-5}$ Among various PAT detection methods, using the single transducer or the ultrasonic array consisting of multiple single transducers are widely implemented. However, the ultrasonics transducer's finite aperture limits the image resolution, ${ }^{6}$ and due to its limited acceptance angle, also limits the field of view (FOV) of the detection system. ${ }^{7}$ In practice, point-like ultrasonic detectors have never been used in PAT because of its poor sensitivity, caused by their high thermal noise level.

Previously, a 2D ring-based virtual point detector was introduced for $\mathrm{PAT},{ }^{8}$ which works like an ideal point detector with high sensitivity. However, the ring-based virtual point detector has a limited imaging region, requiring that the object to be smaller than the ring. Another virtual point detector method, using a positively focused ultrasonic transducer, was previously introduced in ${ }^{9}$ for photoacoustic microscopy (PAM), and its possible application in PAT was also numerically studied. ${ }^{10}$ We extend the second method to include negatively focused transducers, and we are able to construct the high numerical aperture $(\mathrm{NA})(\mathrm{NA}=1)$ transducers to demonstrate their power to be used as virtual point detectors.

\section{HIGH-NA BASED VIRTUAL POINT DETECTORS}

The radiation pattern of a positively focused transducer, at a certain frequency $f$, is shown in Fig. 1. If the transducer's active surface is a part of a spherical shell with a radius of $R$, the focal point $F$ is at the sphere's center. Owing to diffraction, emitted ultrasound waves are not focused to an infinitesimal point at $F$; however, they are focused to a small region with a finite beam diameter (BD). From there, the waves diverge into a region limited by the beam spread angle $\theta_{b}$. From the principle of reciprocity, the radiation pattern also describes the detection pattern.

Further author information: (Send correspondence to Changhui Li) Changhui Li: E-mail: cli@biomed.wustl.edu, Telephone: (314)935-9586

Photons Plus Ultrasound: Imaging and Sensing 2009, edited by Alexander A. Oraevsky, Lihong V. Wang, Proc. of SPIE Vol. 7177, 71770R · ( 2009 SPIE · CCC code: 1605-7422/09/\$18 · doi: 10.1117/12.808514 


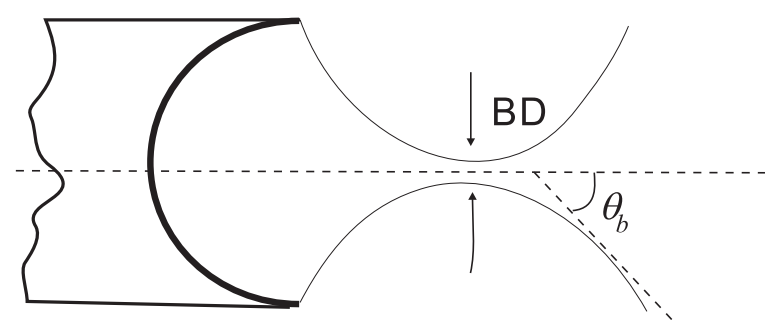

Figure 1. The radiation pattern of a positively focused ultrasonic transducer. The transducer has a spherical curved surface with a radius of $R$, and the focal point $F$ lies at the center of the sphere. The emitted ultrasound is focused to a region with a finite beam diameter (BD). After passing the focal zone, the radiation diverges into a region limited by the beam spread angle $\theta_{b}$.

The beam diameter describes how narrowly the ultrasound can be focused at the focal point, and it is determined by the NA and wavelength. In our previous work, ${ }^{11}$ we calculated the full width at half maximum (FWHM) of the field magnitude at the focal point to represent BD, where the field magnitude at the beam boundary is half of that at the focal point $F$. When the wavelength is much less than the size of the transducer, the amplitude of the emitted field at a vertical location, $\Delta l$ away from the focal point $F$, can be approximately calculated by Rayleigh integral as ${ }^{12}$

$$
\begin{aligned}
\mathrm{A}(\Delta l) & \propto \int_{0}^{\theta_{\max }} \int_{0}^{2 \pi} e^{i k \Delta l \sin (\theta) \cos (\phi)} \sin (\theta) d \theta d \phi \\
& =\int_{0}^{\theta_{\max }} J_{0}(k \Delta l \sin (\theta)) \sin (\theta) d \theta
\end{aligned}
$$

where $\theta, \phi$ are the polar and azimuthal angles, respectively, $J_{0}$ is the Bessel function of the first kind, $k=2 \pi / \lambda$, and $\lambda$ is wavelength. By numerically solving the solution of $|\mathrm{A}(\Delta l)|=|\mathrm{A}(0)| / 2$ (" $|\cdots|$ " represents absolute value), we obtained the relation between $\mathrm{BD}$ and NA. This relation can be approximately fitted by function

$$
\mathrm{BD} \approx 0.62 \frac{\lambda}{\mathrm{NA}} .
$$

Eq. (2) tells us that the beam diameter decreases as the transducer's NA increases. To achieve a "point-like" virtual detector by using the focal point of a positively focused transducer, high-NA transducers are required. In addition, by using a high-NA transducer, the effective size of the virtual point detector is also less than the wavelength, leading to a wide omnidirectional detection angle $\theta_{b}$ over a broad bandwidth.

Similarly, a negatively focused ultrasonic transducer can also be used as a virtual point detector. It differs from a positively focused transducer in that the virtual point detector is located behind the real detection surface. Figure 2(a) represents a virtual point detector by using a positively focused transducer, and (b) is a negatively focused counterpart.

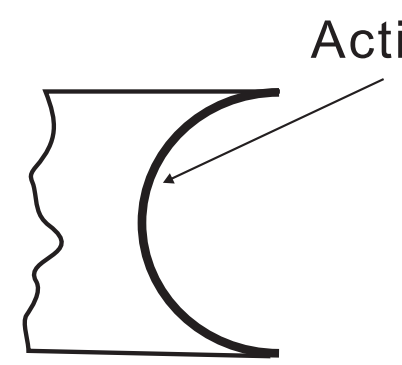

(a)
Active surface

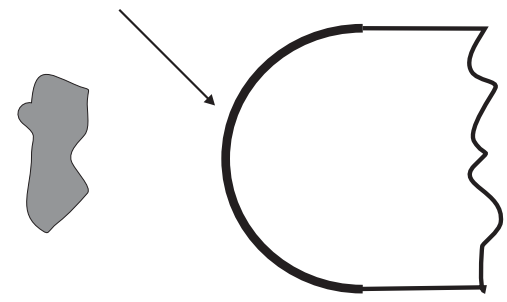

(b)

Figure 2. Two kinds of virtual point detectors. (a) The positively focused transducer; the virtual point is located in front of the detection surface. (b) The negatively focused transducer; the virtual point is located behind the detection surface. 
The impulse response of the 2D virtual point detectors has been approximately described in, ${ }^{8}$ where a steep peak occurs when the wave traveling time reaches the extremum (maximum for positively focused transducer and minimum for negatively focused transducer). Thus, the image reconstruction method used with the virtual point detector is almost the same as the one used with real point detectors, except for minor modifications in the signal time delay. The modifications are, respectively,

- $t=t_{d}+R / c$ for a negative focused virtual point detector, and

- $t=t_{d}-R / c$ for a positive focused virtual point detector,

where $t$ is the time delay used in image reconstructions, $t_{d}$ is the real detection time, $R$ is the radius of the positively or negatively focused ultrasonic transducer, and $c$ is the acoustic speed.

\subsection{Virtual Point Detector Construction}

A metallized polyvinylidene fluoride (PVDF) film (from Measurement Specialties, Inc.), with a thickness of 110 $\mu \mathrm{m}$, was used to construct the transducer. We constructed 2D transducers by bending strips of the film into the half circular shapes seen in Fig. 2. These 2D transducers have NA=1. The PVDF films of the negatively focused and positively focused transducers were all $6.0 \mathrm{~mm}$ in width, and their radii were $12 \mathrm{~mm}$ and $13 \mathrm{~mm}$, respectively. The PVDF films were glued to acrylic plastic surfaces, and the estimated center frequency was around 6.0 MHz. To compare with flat transducers, we further constructed a square flat PVDF transducer, 5.0 $\mathrm{mm}$ by $5.0 \mathrm{~mm}$.

According to this construction and the center frequency, the Rayleigh integral was used to calculate the focal width, ${ }^{12}$ i.e., the effective virtual point detector size, to be around $120 \mu \mathrm{m}$, about half of the wavelength of center frequency. This sub-wavelength virtual detector also has a very broad uniform acceptance angle (close to $\pi / 2$ ), which is another key characteristic of ideal point detectors.

\section{EXPERIMENTS}

\subsection{Phantom Experiments}

The first phantoms were imaged by these virtual point detectors in PAT. As shown in Fig. 3, the PA source contained three black human hair crosses glued on top of optical fibers, with an interval between the hair samples of about $1.0 \mathrm{~cm}$. A Nd:YAG laser (Brilliant B, Quantel) generated $6.5 \mathrm{~ns}, 532 \mathrm{~nm}$ laser pulses with a repetition rate of $10 \mathrm{~Hz}$, which were diverged by a ground glass, so that all three hair crosses were illuminated. Both the phantom and the transducer were immersed in a tank filled with water. The virtual point detectors evenly scanned the object along a horizontal circle, stopping at 240 points, and the signals were averaged of 20 times at each stop. The PA signals were first amplified $40 \mathrm{~dB}$ by a pre-amplifier, and then were recorded by an oscilloscope (Tektronix TDS640A) with a sampling rate of $50 \mathrm{MHz}$. Finally, the recorded signals were sent to a PC for image reconstruction.

The hair phantoms were scanned by the two virtual point detectors and a flat transducer. Fig. 7(a) shows the result of scanning with a positively focused transducer; the scanning radius relative to its focal point was about $4.3 \mathrm{~cm}$. Fig. 7(b) shows the result of scanning with a negatively focused transducer; the scanning radius relative to its focal point was about $4.2 \mathrm{~cm}$. Fig. $7(\mathrm{c})$ shows the result of scanning with a flat transducer; the scanning radius was about $4.2 \mathrm{~cm}$.

Although these three experiments had comparably short scanning distances, the results were clearly different. Both images in Fig. 7(a, b), which were reconstructed from data obtained by two virtual point detectors, presented complete reconstructions with a high and uniform signal-to-noise-ratio (SNR). However in Fig. 7(c), owing to the aperture effect and the limited acceptance angle, the image not only was blurred at the ends of each hair, but also almost completely failed to detect the horizontal hairs on the left and right phantoms.

To alleviate the image blurring and incompleteness, the finite size flat transducer had to be put much further away from the scanning center, at the expense of signal strength. Thus, this phantom experiment demonstrated the advantages of the virtual point detectors over the flat transducer. 


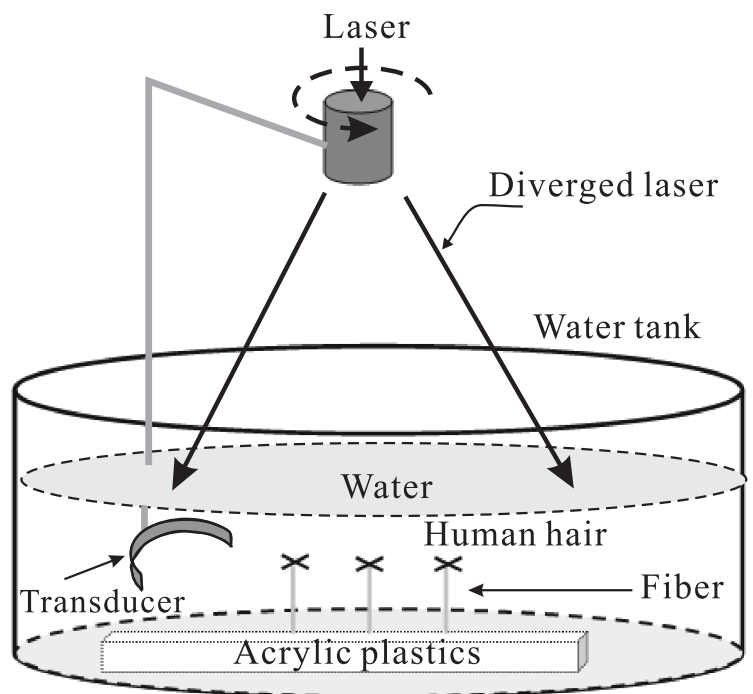

Figure 3. Experimental setup of the phantom experiments by using PAT system.

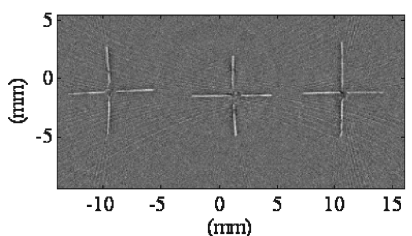

(a)

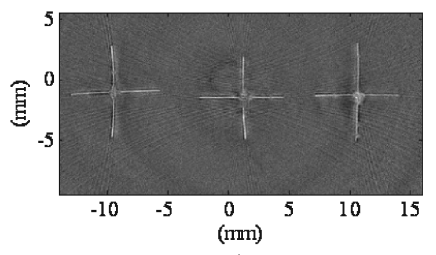

(b)

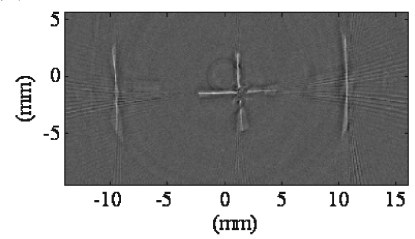

(c)

Figure 4. Reconstructed PAT images. (a) From data gathered by a positively focused PVDF transducer; the scanning radius of the virtual point was $4.3 \mathrm{~cm}$. (b) From data gathered by a negatively focused PVDF transducer; the scanning radius of the virtual point was $4.2 \mathrm{~cm}$. (c) From data gathered by a flat transducer; the scanning radius was $4.2 \mathrm{~cm}$. 
Another phantom experiment is done in TAT system, which replace the laser by a pulsed-microwave source. The source generates $3.0 \mathrm{GHz}$ center frequency, $0.5 \mu$ s pulse width microwave pulses at $100 \mathrm{~Hz}$ repetition rate. The experimental setup is shown in Fig. 5, where the microwave is delivered to the phantom through a horn. Phantoms are made of three low density polyethylene (LDPE) plastic tubes filled with $20 \%$ saline water. Each tube has an outer and an inner diameters of $8 \mathrm{~mm}$ and $6 \mathrm{~mm}$, respectively. The tank is filled with mineral oil that is served as the coupling medium. Ultrasonic detectors scan along a circular trajectory at the horizonal plane, stopping evenly 300 points. The PA signals were first amplified by pre-amplifiers, and then were recorded by an oscilloscope (Tektronix TDS640A). The flat transducer is a commercial 1/4" unfocused transducer with center frequency at $2.25 \mathrm{MHz}$. The positively focused transducer is the same one used in the PAT phantom experiments. The scanning radii of flat and virtual point detectors were $7.6 \mathrm{~cm}$ and $6.4 \mathrm{~cm}$, respectively.

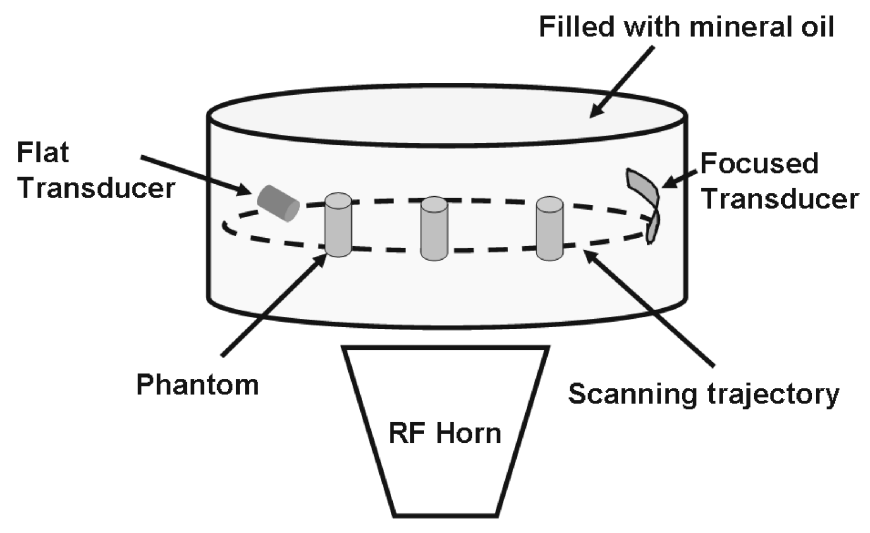

Figure 5. Experimental setup of the phantom experiments by using TAT system.

Figure 6 shows the reconstructed images. Both detectors successfully reconstructed the center tube. However, due to to the limited FOV, the flat transducer failed in reconstructing the two peripheral tubes, resulting in lower SNR and image distortions. On the contrary, the positively focused transducer succeeded in reconstruction both the peripheral tubes.

The TAT primarily intends to detect large tissues, such as the breast. Thus, placing the detectors closer to the tissue can substantially increase the received signal strength, and the detection system can be made more compact.

\subsection{Animal Experiment}

The cerebral cortex of a euthanized Swiss Webster mouse (Harlan Sprague Dawley Incorporated, Indianapolis, Indiana, $\sim 20 \mathrm{~g}$ ) was imaged in situ by PAT. The hair on the mouse head was gently depilated by using a hair removal lotion, then the mouse head was fixed on a homemade animal holder. The experimental setup is similar to the one as shown in Fig. 3, except for that the water tank has a hole in its bottom. The illumination source was a Nd:YAG laser (Brilliant B, Quantel), which generated $6.5 \mathrm{~ns}, 532 \mathrm{~nm}$ laser pulses with a repetition rate of $10 \mathrm{~Hz}$. The illumination laser was homogenized by a ground glass so that the whole cortex region of the mouse could be illuminated. The hole at the bottom of the water tank was sealed by a thin transparent membrane. The mouse head, after a layer of water-based gelatin was applied to it, protruded up into the tank against that membrane. Ultrasonic detectors, either a flat or a high-NA positively focused transducer, were immersed in water at the same horizontal plane as the mouse cortex. Both detectors evenly scanned the cortex along a horizontal circle, stopping at 240 points, and the signals were averaged 20 times at each stop. The PA signals were first amplified $40 \mathrm{~dB}$ by a pre-amplifier, and then were recorded by an oscilloscope (Tektronix TDS640A) with a sampling rate of $50 \mathrm{MHz}$. Finally, the recorded signals were sent to a $\mathrm{PC}$ for image reconstruction. 

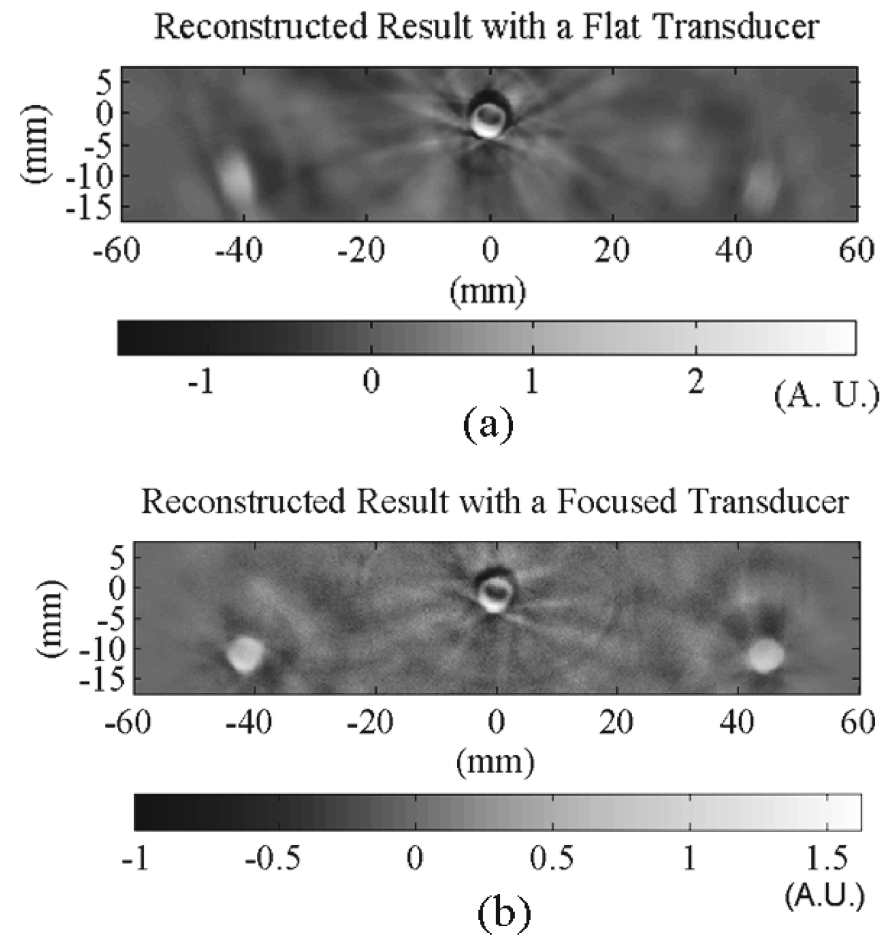

Figure 6. Reconstructed TAT images. (a) From data gathered by a flat transducer; the scanning radius of was $7.6 \mathrm{~cm}$. (b) From data gathered by a positively focused PVDF transducer; the scanning radius was $6.4 \mathrm{~cm}$.

Fig. 7(a) shows the result of scanning with a flat transducer; the scanning radius was about $4.4 \mathrm{~cm}$. Fig. 7 (b) shows the result of scanning with a high-NA-based virtual point detector; the scanning radius was about 2.0 $\mathrm{cm}$. Although the scanning radius of the virtual point detector was shorter than that of the flat transducer, the reconstructed image of Fig. 7(b) exhibits a uniform resolution. Moreover, the reconstructed images of peripheral blood vessels in Fig. 7(a) are blurred. By using a virtual point detector (Fig. 7(b)), the image quality is significantly improved over the image obtained by using a flat transducer (Fig. 7(a)). This result was consistent with the results from phantom experiments in the previous section.

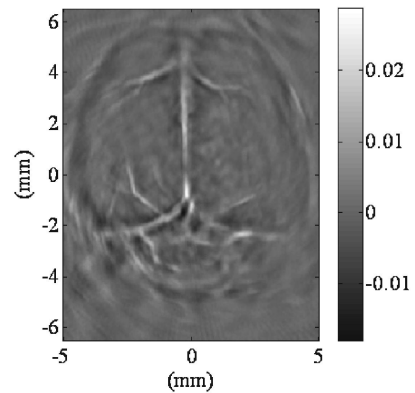

(a)

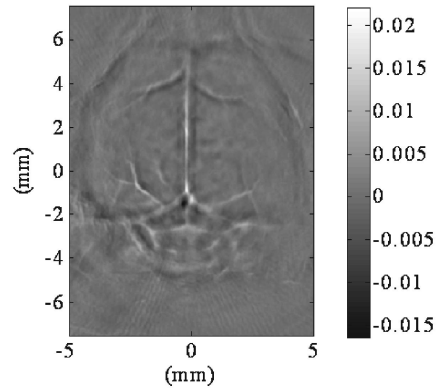

(b)

Figure 7. Reconstructed PAT images. (a) From data gathered by a flat transducer; the scanning radius was $4.4 \mathrm{~cm}$. (b) From data gathered by a positively focused PVDF transducer; the scanning radius of the virtual point was $2.0 \mathrm{~cm}$.

To alleviate the image blurring, the finite size flat transducer had to be placed much further away from the scanning center, at the expense of convenience and signal strength. 


\section{CONCLUSIONS}

In summary, both theoretical analysis and phantom experiments demonstrate that high-NA-based virtual point detectors offer significant improvements over a flat transducer in PAT. Without losing sensitivity relative to flat transducers, virtual point detectors have a negligible aperture effect and a wide acceptance angle, so they can be put close to the PA source, where the signals are strong, maximizing the SNR. Moreover, they can also be used to build an ultrasonic transducer array. In this article, we demonstrated the virtual point detector can be applied both in PAT and TAT. In addition, these virtual point detectors can also be implemented in other tomography methods or technologies that use ultrasonic detectors, such as ultrasonic tomography.

\section{Acknowledgment}

This project was sponsored in part by National Institutes of Health Grant Nos. R01 EB000712, R01 NS46214(BRP), R01 EB000712, and U54 CA136398. L. Wang has a financial interest in Endra, Inc., which, however, did not support this work.

\section{REFERENCES}

[1] $\mathrm{Xu}, \mathrm{M} . \mathrm{H}$. and Wang, L. H. V., "Photoacoustic imaging in biomedicine," Review of Scientific Instruments 77(4), 41101-41122 (2006).

[2] Wang, L. V. and Wu, H.-i., [Biomedical Optics : Principles and Imaging], Wiley, Hoboken, N.J. (2007).

[3] Hoelen, C. G. A., de Mul, F. F. M., Pongers, R., and Dekker, A., "Three-dimensional photoacoustic imaging of blood vessels in tissue," Optics Letters 23(8), 648-650 (1998).

[4] Wang, X. D., Pang, Y. J., Ku, G., Xie, X. Y., Stoica, G., and Wang, L. H. V., "Noninvasive laser-induced photoacoustic tomography for structural and functional in vivo imaging of the brain," Nature Biotechnology 21(7), 803-806 (2003).

[5] Siphanto, R. I., Thumma, K. K., Kolkman, R. G. M., van Leeuwen, T. G., de Mul, F. F. M., van Neck, J. W., van Adrichem, L. N. A., and Steenbergen, W., "Serial noninvasive photoacoustic imaging of neovascularization in tumor angiogenesis," Opt. Express 13(1), 89-95 (2005).

[6] Xu, M. and Wang, L. V., "Analytic explanation of spatial resolution related to bandwidth and detector aperture size in thermoacoustic or photoacoustic reconstruction," Physical Review E 67(5), 056605 (2003).

[7] Li, C., Ku, G., and Wang, L. V., "Negative lens concept for photoacoustic tomography," Physical Review E 78(2), 021901 (2008).

[8] Yang, X. M., Li, M. L., and Wang, L. H. V., "Ring-based ultrasonic virtual point detector with applications to photoacoustic tomography," Applied Physics Letters 90(25), 251103 (2007).

[9] Li, M. L., Zhang, H. F., Maslov, K., Stoica, G., and Wang, L. H. V., "Improved in vivo photoacoustic microscopy based on a virtual-detector concept," Optics Letters 31(4), 474-476 (2006).

[10] Li, M. L. and Wang, L. H. V., "A study of reconstruction in photoacoustic tomography with a focused transducer," in [SPIE International Symposium on Biomedical Optics], 6437, SPIE, San Jose, CA, USA (2007).

[11] Li, C. and Wang, L. V., "High-numerical-aperture-based virtual point detectors for photoacoustic tomography," Applied Physics Letters 3, 033902 (2008).

[12] Pierce, A. D., [Acoustics: An Introduction to Its Physical Principles and Applications], Acoustical Society of America, New York, 1989 ed edition ed. (1989). 